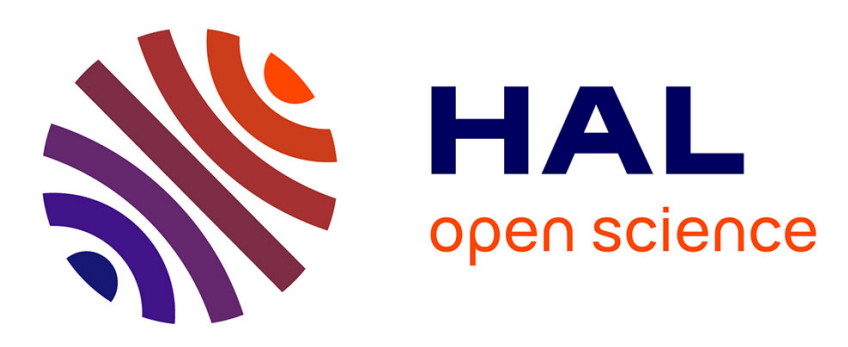

\title{
Total variation and point spread function priors for MLEM reconstruction in Compton camera imaging
}

Yuemeng Feng, Ane Etxebeste, Jean Michel Létang, David Sarrut, Voichita Maxim

\section{- To cite this version:}

Yuemeng Feng, Ane Etxebeste, Jean Michel Létang, David Sarrut, Voichita Maxim. Total variation and point spread function priors for MLEM reconstruction in Compton camera imaging. 2018 IEEE Nuclear Science Symposium and Medical Imaging Conference (NSS/MIC), Nov 2018, Sydney, Australia. 10.1109/NSSMIC.2018.8824767 . hal-02359265

\section{HAL Id: hal-02359265 \\ https://hal.science/hal-02359265}

Submitted on 12 Nov 2019

HAL is a multi-disciplinary open access archive for the deposit and dissemination of scientific research documents, whether they are published or not. The documents may come from teaching and research institutions in France or abroad, or from public or private research centers.
L'archive ouverte pluridisciplinaire HAL, est destinée au dépôt et à la diffusion de documents scientifiques de niveau recherche, publiés ou non, émanant des établissements d'enseignement et de recherche français ou étrangers, des laboratoires publics ou privés. 


\title{
Total variation and point spread function priors for MLEM reconstruction in Compton camera imaging
}

\author{
Yuemeng Feng, Ane Etxebeste, Jean Michel Létang, David Sarrut, Voichiţa Maxim \\ Univ Lyon, INSA Lyon, Université Claude Bernard Lyon 1, UJM-Saint Etienne, CNRS, Inserm, CREATIS UMR 5220, U1206 \\ F69621, Villeurbanne, France \\ yuemeng.feng@creatis.insa-lyon.fr
}

\begin{abstract}
The Compton camera is a gamma ray imaging device already employed in astronomy and still in investigation for clinical domain. A key point in the imaging process is the tomographic reconstruction step. When the acquisition parameters and the a priori information are correctly accounted for, iterative algorithms are able to produce accurate images by compensating for measurement uncertainties and statistical noise. In this work we focus on the list-mode maximum likelihood expectation maximization (LM-MLEM) algorithm with smoothness a priori information expressed by the total variation norm. This type of regularization is particularly well suited for low-dose acquisitions, as it is the case in the applications foreseen for the camera. We show that the TV a priori strongly improves the images when data are acquired in ideal conditions. For realistic data, this a priori is not sufficient and deconvolution with a pre-calculated image-space kernel should also be considered.

Index Terms-Compton camera imaging, gamma ray, maximum likelihood, maximum a posteriori, total variation, point spread function
\end{abstract}

\section{INTRODUCTION}

The number of photons detected by virtual elements of a Compton camera are i.i.d. Poisson random variables. Let $t_{i j}$ denote the probability for a photon emitted in voxel $j$ to be detected as event $i$ and let $\lambda_{j}$ be the mean number of photons emitted in voxel $j$. The contribution of the acquisition uncertainties can be modelled as a convolution in the image domain, in the sense that the data are supposed to come from projections of the real distribution $\lambda$ convolved with some known kernel $k$. With $T=\left(t_{i j}\right)$ denoting the system matrix, the data are thus observations from a multi-variate Poisson law with mean $T(\lambda \otimes k)$. The kernel (also called point spread function, PSF) is a low-pass filter corresponding to various physical effects that occur during the acquisition, e.g. measurement errors, attenuation and scatter. This approach has two relatively opposite effects: on the one hand it brings some smoothness to the solution, on the other hand it improves resolution. The first effect comes from the implicit relaxation of data fidelity constrains. The second is due to the deconvolution capabilities of the MLEM algorithm (known under the name Richardson-Lucy in image processing applications). However the PSF has to be carefully designed in order to

The authors acknowledge financial support of the French National Research Agency through the ANR project 3DCLEAN (ANR-15-CE09-0009), LABEX PRIMES (ANR-11-IDEX-0007) of Université de Lyon and SIRIC LYriCAN Grant INCa-INSERM-DGOS-12563. The first author is supported by a $\mathrm{PhD}$ grant from the China Scholarship Council. capture entirely the data variability without over-corrections that would lead to Gibbs artifacts ( [1]). For instance, for ideal data the PSF should be chosen as a Dirac distribution.

The statistical noise and the ill-conditioned nature of the problem lead to reconstructed images that usually appear overly noisy. As a consequence, the iterations have to be stopped at some point that is difficult to anticipate and the images are usually post-smoothed. Maximum a posteriori expectation maximization (MAP-EM) algorithms have been previously investigated as they may bring information about the source distribution properties and thus regularize the solution. Often smooth priors are considered. In this study we investigate a non-smooth and spatially variant prior, the total variation norm. We show that in conjunction with the use of the image-space PSF, total variation allows to reduce the noise, improve the resolution and accelerate the convergence.

\section{Methods}

\section{A. Image-space PSF deconvolution}

The logarithm of the likelihood of a measurement $Y$ generated from the emission $\lambda$ can be formulated as:

$$
\ln (L(\lambda \mid Y))=\sum_{i}\left(-\sum_{j} t_{i j} \lambda_{j}+y_{i} \ln \left(\sum_{j} t_{i j} \lambda_{j}\right)-\ln \left(y_{i} !\right)\right)
$$

Let $s=\left(s_{j}\right)$ be the sensitivity vector, containing the detection probabilities for photons emitted from voxels $j$. The MLEM algorithm consists in calculating, starting from some initial value $\widehat{\lambda}^{0} \in\left(\mathbb{R}_{+}^{*}\right)^{J}$, the sequence:

$$
\widehat{\lambda}_{j}^{l+1}=\frac{\widehat{\lambda}_{j}^{l}}{s_{j}} \sum_{i} \frac{t_{i j} y_{i}}{\sum_{k} t_{i k} \widehat{\lambda}_{k}^{l}} .
$$

It solves the minimization problem:

$$
\min \left\{-L(\lambda \mid Y): \lambda \in\left(\mathbb{R}_{+}\right)^{J}\right\} .
$$

In the list-mode version that we use in this work, the data space is highly sampled in order to have $y_{i} \in\{0,1\}$.

Image reconstruction and deconvolution can be solved simultaneously with the MLEM algorithm, with two additional convolutions. The algorithm becomes:

$$
\widehat{\lambda}^{l+1}=\frac{\widehat{\lambda}^{l}}{s}(R \otimes k), \quad \text { with } R=T^{T}\left[\frac{1}{T\left(\widehat{\lambda}^{l} \otimes k\right)}\right] .
$$


The image-space PSF method was originally introduced by Snyder et al. [2]. In Reader et al. [3] the method was applied to PET imaging resolution recovery with a Gaussian model for the stationary PSF. Its use for the Compton camera imaging was originally proposed by Kim et al. [4] and was further developed by Jan et al. [5].

\section{B. Total variation regularization}

Since MLEM gives noisy results, a step forward consists in considering regularized solutions of (3) and MAP-EM algorithms. Total variation control is a common method for noise reduction, first intoduced by Rudin et al. [6], which can remove the noise from images while preserving the edges. Adding this regularization to MLEM aims to reconstruct the image with both minimal total variation and maximal likelihood through the resolution of the minimization problem:

$$
\min \left\{-L(\lambda \mid Y)+\alpha G(\lambda): \lambda \in\left(\mathbb{R}_{+}\right)^{J}\right\} .
$$

Here $\alpha \geq 0$ is the weight of TV regularization and $G$ denotes the $L_{1}$-norm of the gradient in the image:

$$
G(\lambda)=\int_{\Omega}|\nabla \lambda(x)| d x .
$$

MAP-EM formalism has already been proposed by Dempster et al. [7]. For the calculation of the MAP-EM estimator with TV prior, Panin et al. [8] proposed a modified version of the MLEM algorithm. However, this solution is unstable and restricted to small values of the smoothing parameter $\alpha$. Other solutions were proposed since then (see, e.g. [9], [10]). In [11], Sawatzky et al. proposed a two-step scheme consisting in smoothing at each iteration the MLEM approximate solution in (2), hereafter denoted by $\hat{\lambda}^{l+1 / 2}$. The smoothing step is a weighted version of the TV denoising algorithm proposed by A. Chambolle [12]. We used a new algorithm for the denoising step proposed by Maxim et al. in [13], based on the dual formulation of the (M) step from the EM algorithm. The (M) step consists in solving:

$$
\widehat{\lambda}^{l+1} \in \underset{\lambda}{\operatorname{argmin}}\{H(\lambda)+\alpha G(\lambda)\},
$$

with

$$
H(\lambda)=\left\langle\lambda-\widehat{\lambda}^{l+1 / 2} \log \lambda, s\right\rangle .
$$

The method is convergent and as fast as the one proposed by Sawatzky et al.

\section{Monte-Carlo simulation}

Monte Carlo simulation of a cylindrical source in vacuum was performed with MEGAlib [14]. The simulation geometry is described in figure 1(a). The camera is composed of silicon strip scatterers (512 strips on each face) and a segmented $\mathrm{LaBr}_{3}$ absorber. The MLEM+TV method was evaluated with ideal data, where only Doppler broadening was accounted for. More realistic data was simulated adding Gaussian uncertainties on the measured energies with $\sigma=1 \mathrm{keV}$ for the scatterers and $\sigma=11.4 \mathrm{keV}$ at $511 \mathrm{keV}$ for the absorber. The position errors are determined by the strip pitch $(\approx 1 \mathrm{~mm})$ and by the

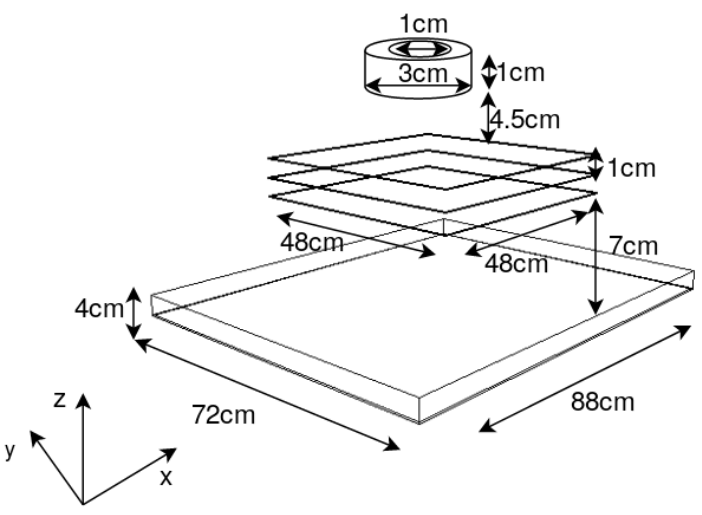

(a) simulation geometry

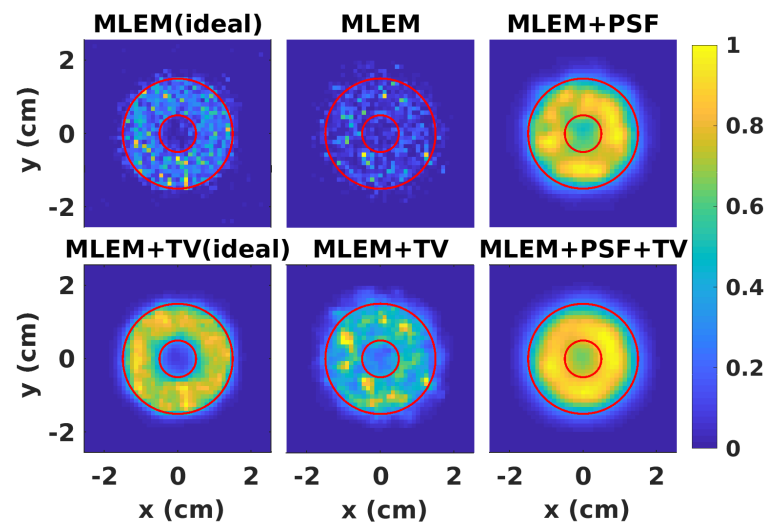

(b) central slices parallel to the detector

Fig. 1. Images reconstructed from ideal data (first column) and realistic data (second and third column). The values of the maximum are normalized to 1 .

size of the crystals $\left(0.5 \times 0.5 \times 4 \mathrm{~cm}^{3}\right)$. The PSF deconvolution is only tested on realistic data. The PSF FWHM is fixed to $16 \mathrm{~mm}$ in $z$ direction and $8 \mathrm{~mm}$ in $x$ and $y$ directions. The reconstructed volume has $(5 \mathrm{~cm})^{3}$ and is divided in $45^{3}$ voxels.

\section{RESULTS AND CONCLUSIONS}

Results from figure 1(b) were obtained from $8 \times 10^{5}$ detected events after 200 MLEM iterations. In the first column, reconstructions for ideal data are shown, with (bottom) and without (top) total variation regularization. The results from the central column show that TV regularization smooths the image, but do not allow to distinguish the hole in the cylinder. The results improve with the use of the PSF (last column).

In this work we focus on Compton camera image reconstruction using realistic data and with low statistics. We show that in this context the image-space PSF deconvolution smooths the result and improves the resolution. TV regularization allows to further smooth the images while reinforcing the edges. We also observed that it promotes convergence. However, the deconvolution did not recover properly the hole since its size is comparable to the FWHM of the system PSF. 


\section{REFERENCES}

[1] S. Stute and C. Comtat, "Practical considerations for image-based PSF and blobs reconstruction in PET," Physics in Medicine \& Biology, vol. 58 , no. 11, p. 3849, 2013.

[2] D. L. Snyder, M. I. Miller, L. J. Thomas, and D. G. Politte, "Noise and edge artifacts in maximum-likelihood reconstructions for emission tomography," IEEE Trans. on Medical Imaging, vol. 6, no. 3, 1987.

[3] A. J. Reader, P. J. Julyan, H. Williams, D. L. Hastings, and J. Zweit, "EM algorithm system modeling by image-space techniques for PET reconstruction," IEEE Trans. on Nuclear Science, vol. 50, no. 5, 2003.

[4] S. M. Kim, H. Seo, J. H. Park, C. H. Kim, C. S. Lee, S.-J. Lee, D. S. Lee, and J. S. Lee, "Resolution recovery reconstruction for a Compton camera," Physics in Medicine \& Biology, vol. 58, no. 9, p. 2823, 2013.

[5] M.-L. Jan, M.-W. Lee, and H.-M. Huang, "PSF reconstruction for Compton-based prompt gamma imaging," Physics in Medicine \& Biology, vol. 63, no. 3, p. 035015, 2018.

[6] L. Rudin, S. Osher, and E. Fatemi, "Nonlinear total variation based noise removal algorithms," Physica D: Nonlinear Phenomena, vol. 60, 1992.

[7] A. P. Dempster, N. M. Laird, and D. B. Rubin, "Maximum likelihood from incomplete data via the EM algorithm," Journal of the royal statistical society. Series B (methodological), pp. 1-38, 1977.

[8] V. Panin, G. Zeng, and G. Gullberg, "Total variation regulated EM algorithm [SPECT reconstruction]," IEEE Transactions on Nuclear Science, vol. 46, no. 6, pp. 2202-2210, 1999.

[9] S. Anthoine, C. Melot, Y. Boursier, and J.-F. Aujol, "Some proximal methods for Poisson intensity CBCT and PET," Inverse problems and Imaging, vol. 6, no. 4, pp. p-565, 2012.

[10] M. Yan, A. A. Bui, J. Cong, and L. A. Vese, "General convergent expectation maximization (EM)-type algorithms for image reconstruction," Inverse Problems \& Imaging, vol. 7, no. 3, pp. 1007-1029, 2013.

[11] A. Sawatzky, C. Brune, F. Wubbeling, T. Kosters, K. Schafers, and M. Burger, "Accurate EM-TV algorithm in PET with low SNR," Nuclear Science Symposium Conference Record, IEEE, 2008.

[12] A. Chambolle, "An algorithm for total variation minimization and applications," Journal of Math. Imaging and Vision, vol. 20, pp. 8997, 2004.

[13] V. Maxim, Y. Feng, H. Banjak, and E. Bretin, "Tomographic reconstruction from Poisson distributed data: a fast and convergent EM-TV dual approach," submitted, https://hal.archives-ouvertes.fr/hal-01892281.

[14] A. Zoglauer, R. Andritschke, and F. Schopper, "MEGAlib-the medium energy gamma-ray astronomy library," New Astron. Rev, vol.50, issues 78, pages 469-648, 2006. 